\title{
Muscle-Tendon Behavior and Kinetics in Gastrocnemius Medialis During Forefoot and Rearfoot Strike Running
}

\author{
Tomonari Takeshita, ${ }^{1}$ Hiroaki Noro, ${ }^{1}$ Keiichiro Hata, ${ }^{1}$ Taira Yoshida, ${ }^{2}$ \\ Tetsuo Fukunaga, ${ }^{1}$ and Toshio Yanagiya ${ }^{1}$ \\ ${ }^{1}$ Juntendo University; ${ }^{2}$ Gifu Pref. Sports Science Center
}

\begin{abstract}
The present study aimed to clarify the effect of the foot strike pattern on muscle-tendon behavior and kinetics of the gastrocnemius medialis during treadmill running. Seven male participants ran with 2 different foot strike patterns (forefoot strike [FFS] and rearfoot strike [RFS]), with a step frequency of $2.50 \mathrm{~Hz}$ and at a speed of $2.38 \mathrm{~m} / \mathrm{s}$ for 45 seconds on a treadmill with an instrumented force platform. The fascicle behavior of gastrocnemius medialis was captured using a B-mode ultrasound system with a sampling rate of $75 \mathrm{~Hz}$, and the mechanical work done and power exerted by the fascicle and tendon were calculated. At the initial contact, the fascicle length was significantly shorter in the FFS than in the RFS $(P=.001)$. However, the fascicular velocity did not differ between strike patterns. Higher tendon stretch and recoil were observed in the FFS $(P<.001$ and $P=.017$, respectively) compared with the RFS. The fascicle in the positive phase performed the same mechanical work in both the FFS and RFS; however, the fascicle in the negative phase performed significantly greater work in the FFS than in the RFS $(P=.001)$. RFS may be advantageous for requiring less muscular work and elastic energy in the series elastic element compared with the FFS.
\end{abstract}

Keywords: foot strike pattern, ultrasound, muscle-tendon interaction, mechanical work

Generally speaking, runners use their preferred foot strike pattern, classified into forefoot strike (FFS), midfoot strike, and rearfoot strike (RFS). ${ }^{1}$ Previous researchers have investigated the effect of foot strike patterns on the running performance from the viewpoint of biomechanical variables. This has been especially true of the FFS, as it induces a reduced braking and impact force during stance phase compared with the RFS. ${ }^{2}$ However, most recreational runners, in addition to elite marathon runners, ${ }^{3}$ land each stride on their heels in a long-distance race. ${ }^{1,4}$ In fact, some studies have observed reduced energy expenditure in the RFS compared with the FFS or MFS at the same running speed..$^{5,6}$ Therefore, it is crucial to clarify the effect of foot strike patterns on running performance.

In the last 2 decades, studies have revealed both kinetic and kinematic differences between FFS and RFS running. For example, reduced knee flexion and ankle dorsiflexion were observed in the stance phase of FFS running. ${ }^{7-9}$ In addition, a greater negative ankle joint moment was generated in the first half of the stance phase of FFS running, rather than RFS running. ${ }^{10}$ In the first half of the stance phase, while running, the muscle-tendon units (MTU) of the ankle plantar flexors are lengthened, whereas they are shortened in the second half of the stance phase. ${ }^{11,12}$ It is therefore considered that elastic energy is stored in the MTUs of plantar flexors due to the negative ankle joint moment during the early stance, and this energy is then released in the latter half of the stance phase. The use of elastic energy in the MTU of the lower limb was thought to be a

Takeshita, Noro, Hata, Fukunaga, and Yanagiya are with the Graduate School of Health and Sports Science, Juntendo University, Chiba, Japan. Takeshita, Noro, Hata, and Yanagiya are also with the Institute of Health and Sports Science \& Medicine, Juntendo University, Chiba, Japan. Yoshida is with the Gifu Pref. Sports Science Center, Gifu, Japan. Yanagiya (tyanagi@juntendo.ac.jp) is corresponding author. way to reduce metabolic energy expenditure while running. ${ }^{10,13}$ However, a different study revealed that the net work of the ankle joint was higher in the FFS than in the RFS at the same running speed. ${ }^{14}$ Excessive mechanical work (ie, excessive muscular work) in the FFS would make the FFS less economical than the RFS. To better understand the relationship between the foot strike pattern and energy expenditure, it is necessary to evaluate the mechanical work done by plantar flexors during the stance while running in vivo.

Some previous studies revealed both the muscle and tendon behavior during different foot strike running. Swinnen et $\mathrm{al}^{15}$ reported that fascicle shortening velocity of the gastrocnemius medialis (GM) while running was higher in RFS runners rather than in FFS runners. In addition, Suzuki et $\mathrm{al}^{16}$ demonstrated that GM fascicle length was longer in the RFS than the FFS throughout the gait cycle. While the difference in muscle-tendon kinematics has been investigated between the FFS and RFS, there is little information regarding muscle-tendon kinetics with consideration to the foot strike pattern while running. Based on computer simulations, only Yong et $\mathrm{al}^{17}$ have reported higher negative work in the GM fascicle while FFS running compared with RFS running. They also revealed there was no significant difference in tendon work between FFS and RFS running. ${ }^{17}$ However, estimations of muscle-tendon behaviors by computer simulation and by in vivo experiments are different. For instance, Yong et al ${ }^{17}$ observed higher lengthening of the GM fascicle during stance in RFS than in FFS running. Conversely, several studies concluded that the GM fascicle is not lengthened during stance when runners select the FFS. ${ }^{15,16}$ This difference may affect the interpretation of mechanical work done by both the muscle and tendon. Since previous studies revealed no significant difference in maximum running velocity between FFS and RFS runners, ${ }^{15}$ both positive and negative work by the GM muscle would not be different between the foot strike patterns. Considered together with the 
previous finding that ankle joint work was higher in the FFS than in the RFS in the stance phase, ${ }^{14}$ mechanical work by the GM tendon during stance would be lower in RFS running than in FFS running.

This study aimed to investigate the differences in musculotendinous behavior and kinetics of the GM between FFS and RFS running. We hypothesized that (1) the length of the GM fascicle in the stance phase would be shorter in FFS than RFS running, but both positive and negative work of the GM fascicle would not be different between the foot strike patterns, (2) the stretch and recoil of tendinous tissue would be higher in FFS than RFS running, and (3) both positive and negative mechanical work would be higher in FFS than RFS running. These musculotendinous kinematics and kinetics would provide us with further information on whether runners should select the FFS or RFS pattern.

\section{Methods}

Seven healthy (mean $[\mathrm{SD}]=$ age, 22.4 [0.7] y; body height, 1.76 [0.07] m; body mass, 67.1 [2.2] kg) male runners who trained at least 4 times per week participated in the present study. The participants had no history of running-related injury or pain in their lower limbs for $>6$ months. All participants gave written informed consent for participation in this study. All procedures were conducted according to the Declaration of Helsinki and were approved by the local ethical committee (Health and Sports Science Ethics Committee of Juntendo University, project identification code: 30-27).

Each participant ran on an instrumented treadmill (FTMH1244; Tec Gihan Co, Ltd, Kyoto, Japan) on 2 different days. On the first day, the participants had a prerunning trial on the treadmill at the speed of $2.38 \mathrm{~m} / \mathrm{s}(8.57 \mathrm{~km} / \mathrm{h})$ for more than 5 minutes, barefoot, with their preferred step frequency. We collected the ground reaction force $(\mathrm{GRF})$ of the prerunning trial (sampling rate $1 \mathrm{kHz}$ ) from the force platform embedded under the treadmill. The participants' step frequency was determined from the duration between the initial contact and the next contact of the opposite foot.

On the second day, the participants performed a warm-up, both shod and barefoot, running on a treadmill for $>5$ minutes at the same speed as that of the prerunning trial. We checked their habitual foot strike pattern during barefoot warm-up running based on a previous study, ${ }^{1}$ and there were 5 FFS, 1 midfoot strike, and 1 RFS runner. During the experimental trials, they ran on the treadmill twice for 45 seconds at a speed of $2.38 \mathrm{~m} / \mathrm{s}$. During the experimental trials, we instructed the participants to run with FFS (one trial) and RFS (different trial) and to adjust their step frequency to $2.5 \mathrm{~Hz}$ with an electrical metronome. This experimental step frequency was set as the mean of their habitual step frequency measured on the first day. More than 3 minutes of rest was provided between trials. The order of the foot strike patterns was randomized across participants. The participants' foot strike pattern was recorded in the sagittal plane using a high-speed video camera (240 fps; LUMIX DMC-FZ300; Panasonic, Osaka, Japan) A successful trial was defined as occurring when the participants performed the prescribed foot strike pattern.

The kinematic data of the left leg were obtained from 5 retroreflective markers (placed on the great trochanter, knee joint, malleolus, calcaneus, and second metatarsal phalangeal joint) using a motion capture system with 13 cameras $(250 \mathrm{~Hz}$; VICON Nexus, version 2.3; VICON, Oxford, United Kingdom) installed beside the treadmill. The horizontal plane (both kinematics and kinetics) was parallel with the treadmill belt. There was no incline or decline in the treadmill. The coordinate data of each marker were smoothed with a fourth-order low-pass Butterworth filter with a cutoff frequency of $6 \mathrm{~Hz}$. From the smoothed data, the left-leg knee and ankle joint angles were calculated in the sagittal plane (Figure 1). Knee joint angle was defined as the external angle between the thigh and tibia, and the ankle joint angle was defined as the external angle between the tibia and foot (defined as the line from the heel to the second metatarsal joint). Finally, the foot strike angle, defined as the angle between the foot and ground, was calculated in the sagittal plane to observe how participants land at initial ground contact. All kinematic analyses were conducted using a custom-made MATLAB script (MathWorks Inc, Natick, MA).

The GRFs and moments were recorded from the force platform embedded under the treadmill with a sampling frequency of $1 \mathrm{kHz}$. The raw data were imported to a computer and filtered with a fourth-order low-pass Butterworth filter with a cutoff frequency of $20 \mathrm{~Hz}$ using a custom-made MATLAB script. The threshold for foot contact was set at $50 \mathrm{~N}$ to help determine the stance phase of each gait cycle. We then calculated joint kinetics from the combination of joint kinematics and GRFs. The moment about the ankle was calculated using inverse dynamics in the sagittal plane. ${ }^{18}$ The length of the Achilles tendon moment arm was estimated using coefficient and joint angles. ${ }^{19}$ The Achilles tendon force was estimated as the ratio of the ankle joint moment to the instantaneous length of the Achilles tendon moment arm. Ankle joint power was calculated as the product of the joint moments and angular velocities, and joint work (both negative and positive) was calculated as the time integral of the ankle joint power curve. Ankle joint power and work were normalized to the participants' body mass.

Recorded GRF data were also used to determine the spatialtemporal parameters of the running pattern. Contact time was calculated as the duration of over $50 \mathrm{~N}$ vertical GRF of the left foot. Flight time was defined as the time from the left foot toe-off to the touchdown of the right foot. The ratio of the contact time to the total time of one gait cycle was calculated. Step time was evaluated as the sum of the contact time and flight time, and then step frequency was calculated as the reciprocal of the step time. From the ratio of the step frequency to the treadmill belt speed, the step length was calculated for each step.

A custom-ordered $7.5 \mathrm{MHz}$ linear array probe $(7 \mathrm{~cm}$ width) was placed on the muscle belly of the GM and aligned with the midline of the muscle using adhesive tape to observe GM muscle behavior. Real-time ultrasound images of the GM fascicles were recorded using ultrasound with a sampling frequency of $75 \mathrm{~Hz}$ (SSD-4000; ALOKA, Tokyo, Japan). GM fascicle tracking was manually conducted for 5-step cycles using motion analysis software (Frame-DIAS V; DKH, Tokyo, Japan). In each ultrasound image, we digitized both fascial and deep cross points of the fascicle and tendinous tissue. GM fascicle length $\left(L_{\text {Fascicle }}\right)$ was calculated as the length between both fascial and deep cross-points. Simultaneously, the GM fascicle pennation angle was calculated as the angle between the fascicle and the deep aponeurosis. This digitization process was conducted twice to evaluate the reliability of this fascicle length measurement, and the coefficient for variation was below 3\%. The entire MTU length in GM ( $\left.L_{\mathrm{MTU}}\right)$ was estimated using both the knee and ankle joint angles in the sagittal plane, and regression equations. ${ }^{20}$ The length of the series elastic element $\left(L_{\mathrm{SEE}}\right)$ of the GM was estimated from the equation using $L_{\mathrm{MTU}}, L_{\text {Fascicle, }}$, and the pennation angle. ${ }^{21}$ That is,

$$
L_{\mathrm{SEE}}=L_{\mathrm{MTU}}-L_{\text {Fascicle }} \cdot \cos \theta,
$$

where $\theta$ indicates the instantaneous pennation angle of the GM fascicle. The above variables were increased to $1 \mathrm{kHz}$ with cubic spline interpolation. The contraction velocity of each tissue was 


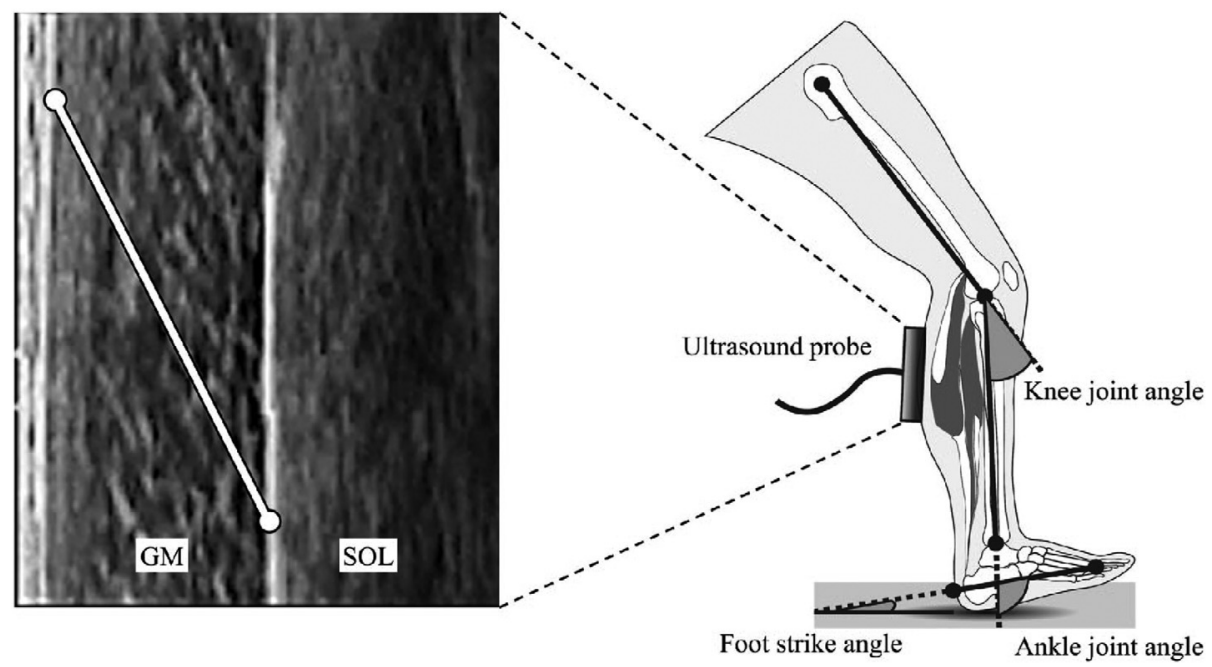

Figure 1 - Example of an ultrasound image of the gastrocnemius and the definition of the kinematic variables (knee joint, ankle joint, and foot strike angle) in the sagittal plane during running trials. Each joint angle was defined as the exterior angle between segments. GM indicates gastrocnemius medialis; SOL, soleus.

calculated relative to time, and a concentric contraction was defined as a positive contraction velocity.

The force of the SEE attributed to the GM was estimated from the product of the Achilles tendon force and the relative physiological cross-sectional area of the GM (assumed to be $0.159^{22}$ ). The fascicle force of the GM was calculated as the ratio of SEE force to the instantaneous cosine of the GM fascicle. Mechanical power was calculated as the product of the tensile force and contraction velocity of the MTU, fascicle, and SEE. ${ }^{23}$ Both positive and negative work were calculated from the integration of each tissue's power curve relative to time. Mechanical power and work done by each tissue was normalized to the participants' body mass and averaged for each foot strike pattern.

To evaluate the GM muscle activity while running, we used a multichannel telemeter system (WEB-7000; Nihon Koden, Tokyo, Japan). An electromyogram sensor (ZB-150 H; mass $=10 g$, electrode distance $=10 \mathrm{~mm}$, sampling rate $=1 \mathrm{kHz}$ ) was attached with adhesive tape to the muscle belly of the right GM so as to not interfere with the ultrasound probe placement. The collected data were digitized through an analog to digital converter (PowerLab; ADInstruments, Sydney, Australia). These data were filtered to eliminate motion artifact (fourth-order, zero-phase-lag band-pass filter, 20-450 Hz). Then, the filtered data were full-wave rectified and smoothed (lowpass, fourth-order, zero-phase-lag Butterworth filter with $6 \mathrm{~Hz}$ cutoff frequency). In addition, the participants had another plantar flexion movement on the standing position as maximum voluntary contraction. At that time, we added hand-to-hand resistance on their shoulder, taking care not to generate motion in other joints. The amplitude of the filtered signal from the GM muscle was normalized to the value of the maximum voluntary contraction.

Electromyogram signals, kinematics, kinetics, and ultrasound data were synchronized using an analog input for the start of the ultrasound image recording. All parameters were evaluated and averaged for 5 gait cycles and expressed as mean (SD). A paired $t$ test was used to test the significance of between-condition differences in all variables. Furthermore, an a priori statistical analysis was performed for all variables to examine the effect size and statistical power, based on the mean difference and SD between the 2 -foot strike patterns. The above statistical analysis was performed with R software (version 3.5.2; R Development Core Team, Vienna, Austria). The level of significance was set to $\alpha=.05$.

\section{Results}

Regarding the foot strike angle, there was a significant difference between the RFS and FFS $(P<.001)$. All other spatiotemporal parameters indicated no difference between the FFS and RFS (Table 1).

Both knee and ankle kinematics were different between the FFS and RFS during stance. Switching to the FFS resulted in more ankle plantar flexion at initial contact, maximum flexion, and toe-off compared with the RFS (Table 2; $P<.001, P=.004$, and $P=.004$, respectively). There was no significant difference in the knee joint angle at the initial contact and toe-off between the FFS and RFS (Table $1 ; P=.697$ and $P=.527$, respectively). The maximum knee flexion tended to be higher in the FFS than in the RFS $(P=.076)$.

The behavior of each MTU, fascicle, and SEE was different between the FFS and RFS in some points during stance (Figure 2C-2E; Table 3 ). At the initial contact, $L_{\mathrm{MTU}}, L_{\text {Fascicle }}$, and $L_{\mathrm{SEE}}$ were significantly longer in the RFS than in the FFS $(P<.001, P=.001$, and $P=.006$, respectively). Nevertheless, there was no significant difference in the amount of fascicle shortening, fascicular average velocity, or maximum fascicular velocity between RFS and FFS running $(P=.281, P=.970$, and $P=.665$, respectively). The maximum $L_{\mathrm{MTU}}$ was significantly greater in the RFS than in the FFS $(P=.001)$, while the maximum $L_{\mathrm{SEE}}$ was greater in the FFS than in the RFS $(P=.017)$. Finally, SEE stretch and recoil were significantly greater in the FFS than in the RFS $(P<.001$ and $P=.017$, respectively).

Regarding muscle and tendon kinetics, the maximum GM muscle force was significantly higher in the FFS than in the RFS (15.87 [1.39] N/kg in the FFS and 12.91 [1.31] N/kg in the RFS, $P=.010)$, and the same trend was observed in the SEE (13.68 $[1.01] \mathrm{N} / \mathrm{kg}$ in the FFS and $11.53[1.26] \mathrm{N} / \mathrm{kg}$ in the RFS, $P=.020)$. Examining the mechanical work (Figure 3), positive fascicle work tended to be higher in the FFS than in the RFS, but there was no significant difference $(P=.090)$. However, fascicular negative 
Table 1 Results of Foot Strike Angle and Spatial-Temporal Variables Between FFS and RFS Running

\begin{tabular}{lcccc}
\hline Variables & FFS & RFS & P & ES \\
\hline Foot strike angle, deg & $-9.9(2.5)$ & $7.0(2.0)$ & $<.001$ & 11.764 \\
Contact time, ms & $246.3(15.6)$ & $254.1(19.2)$ & .400 & 1.244 \\
Flight time, ms & $153.1(15.4)$ & $142.0(18.3)$ & .165 & 1.000 \\
Step frequency, step/s & $2.50(0.01)$ & $2.53(0.01)$ & .220 & 0.782 \\
Step length, m/step & $0.95(0.00)$ & $0.94(0.00)$ & .217 & 1.486 \\
\hline
\end{tabular}

Abbreviations: ES, effect size; FFS, forefoot strike running; RFS, rearfoot strike. Note: Variables are shown as the mean (SD) in the respective column of each foot strike pattern. In all rows, the difference in each variable between FFS and RFS is calculated. Statistical results, such as $P$ value, ES, and power, are described in all rows.

Table 2 Results of Kinematic Variables in Knee and Ankle Joint Between FFS and RFS Running in Stance Phase

\begin{tabular}{|c|c|c|c|c|c|}
\hline Variables & FFS & RFS & $P$ & ES & Power \\
\hline \multicolumn{6}{|l|}{ Knee joint } \\
\hline At initial contact, deg & $24.4(3.5)$ & $23.9(2.2)$ & .697 & 2.286 & 1.000 \\
\hline Maximum flexion, deg & $45.3(5.5)$ & $47.9(3.8)$ & .076 & 1.115 & 0.830 \\
\hline At toe-off, deg & $24.1(3.4)$ & $24.5(4.6)$ & .527 & 1.420 & 0.950 \\
\hline \multicolumn{6}{|l|}{ Ankle joint } \\
\hline At initial contact, deg & $83.5(3.5)$ & $98.7(2.6)$ & $<.001$ & 4.759 & 1.000 \\
\hline Maximum flexion, deg & $108.8(1.8)$ & $111.4(1.0)$ & .004 & 1.889 & 0.997 \\
\hline At toe-off, deg & $69.8(3.7)$ & $76.5(4.4)$ & .004 & 1.873 & 0.996 \\
\hline
\end{tabular}

Abbreviations: ES, effect size; FFS, forefoot strike; RFS, rearfoot strike. Note: Variables are shown as the mean (SD) in the respective column of each foot strike pattern. In all rows, the difference in each variable between FFS and RFS is calculated. Statistical results, such as $P$ value, ES, and power, are described in all rows.

work was significantly greater in the FFS than in the RFS ( $P=$ .001). Negative mechanical work done by MTU was significantly higher in the FFS than in the RFS $(P=.020)$, and a similar but insignificant trend was present for the $\operatorname{SEE}(P=.100)$. In contrast to the difference in negative work, the positive work by MTU was higher in the FFS than in the RFS $(P=.001)$, and a similar result was seen in the SEE $(P<.001)$.

\section{Discussion}

The present study aimed to investigate fascicle-tendon behavior characteristics and GM muscle kinetics between FFS and RFS running. These results suggest that FFS running requires a shorter fascicular length and greater SEE elongation during the stance phase. To the best of our knowledge, this is the first study that demonstrates differences in the behavior and kinetics of GM musculotendinous tissues between the FFS and RFS.

Our first hypothesis was that the GM fascicle would be shorter in FFS compared with RFS running. The $L_{\mathrm{FAS}}$ at the initial contact was shorter during FFS running than RFS running (Table 3). Interestingly, the GM fascicle maintained a shorter length throughout the stance phase in the FFS compared with the RFS (Figure 2C), and we suggest that GM activity in the late flight phase affects the behavior of the GM fascicle after initial ground contact. Based on the force-length relationship within the sarcomere, previous studies have reported that shorter fascicle length is disadvantageous to generate force while running. ${ }^{24}$ Considering the results of the present study, we can argue that the shorter fascicular length during the FFS may be a factor in the large energy expenditure in FFS running reported by previous studies. ${ }^{5,13}$ In addition, it has been reported that FFS running produces greater GRF due to the longer GRF moment arm than RFS running. ${ }^{25}$ In addition to greater GRF, we observed higher fascicle and SEE force in the FFS than the RFS. While FFS running, runners may be required to generate higher GM muscular force to support and move their body forward. In combination with the results of previous studies, we observed that the FFS is disadvantageous to generate greater fascicle force in the GM, due to both the shorter sarcomere length and the longer GRF moment.

There was no difference in the positive work done by the GM fascicle, but there was in the negative work. Since the step frequency was controlled, force and contraction velocity are the main factors to effect mechanical work. Previous studies reported that higher GM muscular force and lower contraction velocity were seen in FFS than RFS during stance. So, we hypothesized there would be no difference in both positive and negative work done by the GM fascicle during stance between the FFS and RFS because it would be counterbalanced by multiplying the muscular force and velocity. During the negative phase, the GM fascicle produced higher mechanical work in the FFS compared with the RFS (Figure 3B). Based on computer simulation, Yong et $\mathrm{al}^{17}$ reported a similar tendency, though they reported a higher amount of mechanical work. Their simulation study reported notable lengthening of the GM fascicle after the initial contact in the FFS; however, in our in vivo study, there was little change in the GM fascicle length throughout the stance phase both in the FFS and RFS. During stance, the GM fascicle length remained almost the same, and the mechanical work on the fascicle was markedly less than that of the SEE, in both the FFS and RFS. Therefore, fascicular mechanical work might not be considered in running economy at such slow running speeds.

In the last hypothesis, we thought higher mechanical work would be seen in the SEE during stance following the higher amount of stretch and recoil in the FFS than the RFS. Similar to previous work, while FFS running, both the entire MTU and SEE demonstrated greater lengthening in the first half of the stance phase (Figure 2D and 2E). Mechanical work in previous research ${ }^{15}$ has implied that a greater amount of elastic energy in MTU would 

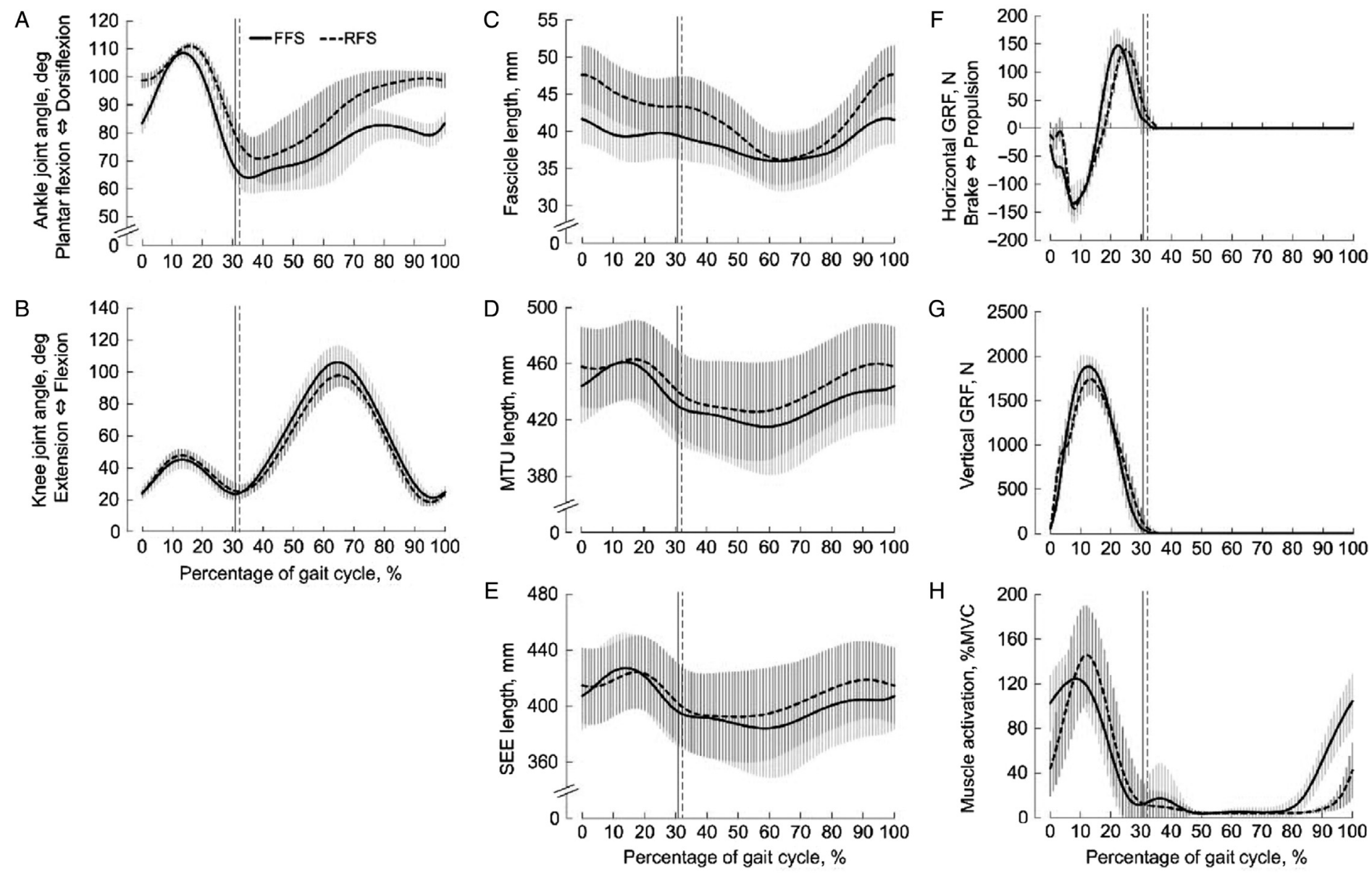

Figure 2 - Ensemble mean time series data $(\mathrm{n}=7)$ during one gait cycle with FFS (solid) and RFS (dashed). (A) Ankle angle, (B) knee angle, (C) GM fascicle length, (D) entire MTU length, (E) SEE length, (F) horizontal GRF, (G) vertical GRF, and (H) GM muscle activation (\%MVC) with respect to the percentage of one gait cycle. The solid vertical line indicates the timing of toe-off in FFS running, and the dashed one indicates that in RFS running. \% MVC indicates maximum voluntary contraction; FFS, forefoot strike; GM, gastrocnemius medialis; GRF, ground reaction force; MTU, muscle-tendon units; RFS, rearfoot strike; SEE, series elastic element.

Table 3 Results of Muscle-Tendon Behavior Between FFS and RFS Running in Stance Phase

\begin{tabular}{|c|c|c|c|c|c|}
\hline Variables & FFS & RFS & $P$ & ES & Power \\
\hline \multicolumn{6}{|l|}{ Muscle-tendon unit } \\
\hline At initial contact, mm & $444.1(26.3)$ & $457.9(28.1)$ & $<.001$ & 5.224 & 1.000 \\
\hline Maximum, mm & $461.5(27.3)$ & $463.4(27.4)$ & .001 & 2.764 & 1.000 \\
\hline Stretch, mm & $17.3(2.8)$ & $5.5(1.9)$ & $<.001$ & 2.852 & 1.000 \\
\hline Recoil, mm & $29.5(3.3)$ & $25.9(3.9)$ & .074 & 5.572 & 1.000 \\
\hline \multicolumn{6}{|l|}{ Fascicle } \\
\hline At initial contact, $\mathrm{mm}$ & $41.7(3.3)$ & $47.6(3.9)$ & .001 & 2.571 & 1.000 \\
\hline Shortening, mm & $4.1(0.9)$ & $5.2(2.2)$ & .281 & 1.114 & 0.692 \\
\hline Average velocity, $\mathrm{mm} / \mathrm{s}$ & $24.5(5.7)$ & $24.7(7.4)$ & .970 & 1.274 & 0.906 \\
\hline Maximum velocity, $\mathrm{mm} / \mathrm{s}$ & $47.7(15.4)$ & $51.9(15.0)$ & .665 & 1.344 & 0.840 \\
\hline \multicolumn{6}{|l|}{ Series elastic element } \\
\hline At initial contact, $\mathrm{mm}$ & $407.3(24.1)$ & $414.8(26.7)$ & .006 & 1.715 & 0.963 \\
\hline Maximum, mm & $427.6(24.8)$ & $424.6(25.8)$ & .017 & 1.561 & 0.927 \\
\hline Stretch, mm & $20.3(1.7)$ & $9.8(3.5)$ & $<.001$ & 3.104 & 1.000 \\
\hline Recoil, mm & $29.9(3.6)$ & $25.1(3.4)$ & .017 & 1.666 & 0.953 \\
\hline
\end{tabular}

Abbreviations: ES, effect size; FFS, forefoot strike; RFS, rearfoot strike. Note: Variables are shown as the mean (SD) in the respective column of each foot strike pattern. In all rows, the difference in each variable between FFS and RFS is calculated. Statistical results, such as $P$ value, ES, and power, are described in all rows. 

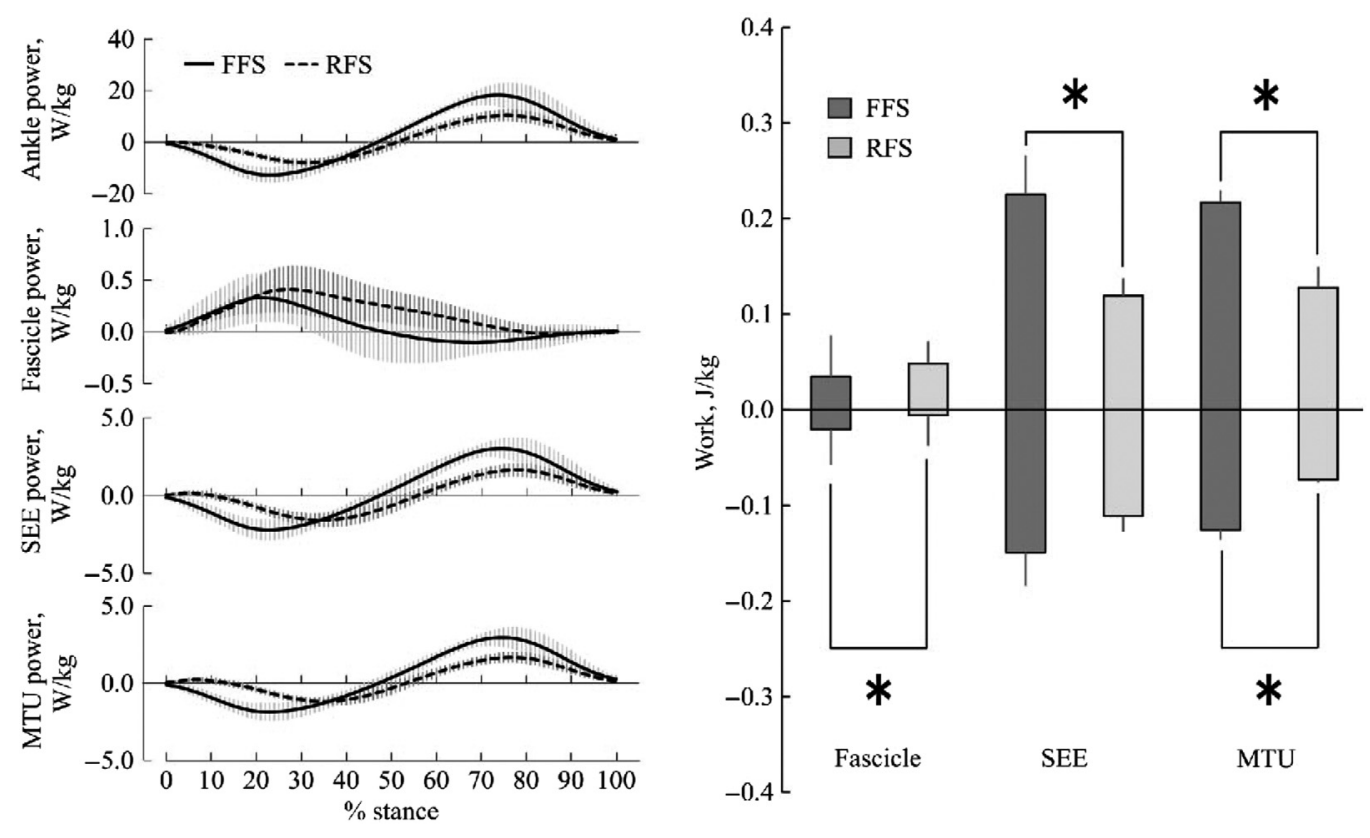

Figure 3 - The mean and SD of mechanical variables done by fascicle, SEE, and MTU in the stance phase $(n=7)$. (A) Mean time series data of mechanical power done by ankle joint, fascicle, SEE, and MTU of the GM in the stance phase. The solid line indicates the result of FFS, and the dashed line indicates the result of RFS. All data were normalized to 101 points over an entire stance. (B) Normalized mean value and SD of mechanical work done by each tissue in the stance phase. The dense gray scale indicates FFS, and the light gray scale indicates RFS. FFS indicates forefoot strike; GM, gastrocnemius medialis; MTU, muscle-tendon units; RFS, rearfoot strike; SEE, series elastic element. *Significant difference between FFS and RFS $(P<.05)$.

be stored during FFS runners than RFS runners, and there would be a higher release of this energy in FFS runners. In our findings, both the MTU and SEE of the GM were not lengthened during the RFS from the initial contact to about $5 \%$ of the gait cycle duration. In this phase, the first peak of vertical ground reaction force was generated in the RFS (Figure 2G). These results indicate that storage of elastic energy from the negative plantar flexion moment and power in the ankle joint did not occur in the RFS in the first half of stance. ${ }^{14,26}$ In our study, both the stretch and recoil of the SEE in the GM were greater in FFS running than in RFS running (Table 1), whereas, Swinnen et al ${ }^{15}$ reported there was no significant difference in SEE behavior between FFS runners and RFS runners. One of the factors contributing to this difference in SEE recoil may be the participants' individual tendon stiffness. Arampatzis et $\mathrm{al}^{27}$ demonstrated a negative relationship between Achilles tendon stiffness and energy consumption during endurance running. Because many faster runners perform FFS during athletics races, ${ }^{1}$ it is considered that FFS runners have a stiffer Achilles tendon and that is why FFS runners can run faster than RFS runners. However, to the contrary, Kubo et $\mathrm{al}^{28}$ observed no significant difference in Achilles tendon stiffness between habitual FFS and RFS runners. These previous studies indicate that there is no unified perspective on the effect of tendinous properties and foot strike pattern on energy consumption while running. We conclude that FFS is superior in generating higher force than RFS due to the greater positive and negative work done by MTU in the stance phase, but it is still unknown whether the mechanical property of the SEE affects the habitual foot strike pattern. Future studies are needed to clarify whether the mechanical properties of muscle and tendon affect actual behavior and economy during running, considering foot strike patterns.

In this study, we considered the behavior of muscles and tendons in different foot strike patterns, which may affect running performance. Some studies have reported that, with the FFS, higher
Achilles tendon loading occurs during stance, compared with the RFS, ${ }^{29-31}$ and they warned of FFS injuries, such as Achilles tendinopathies. On the other hand, habitually, RFS runners have twice the rate of the repetitive stress injuries than habitual FFS runners. ${ }^{32}$ While runners can benefit from the performance-related benefits of the FFS and RFS (ie, obtaining spring-like elasticity in the FFS and less muscular mechanical energy in the RFS), they may be exposed to the risk of injury that is unique to each. Since runners can convert their foot strike pattern while running, it may be suitable to use both foot strike patterns according to distance and the situation for improving performance and preventing running injury.

A limitation of the present study is that we did not consider the behavior of the soleus muscle. As running speed increases, the use of soleus during stance increases, and hence, gastrocnemius activity did not increase. ${ }^{33}$ It would be useful to consider the behavior of both the gastrocnemius and soleus fascicle to understand the energy expenditure of the triceps surae during running. Moreover, we only tested one running speed, making our results applicable only to that pace. This speed was drastically slower than that of elite longdistance races. However, this speed was valuable to clarify the behavior and energetics in both fascicles and the SEE during FFS running compared with RFS running. Some studies have suggested that muscle-tendon interaction is altered by running speed, and greater fascicle concentric activation was observed at higher running speeds. ${ }^{12,15,16}$ There was little information available for the comparison of fascicle and SEE kinetics between foot strike patterns while controlling some factors, such as step frequency, during in vivo running. Future studies are needed to reveal the actual effect of foot strike pattern on muscle-tendon behavior and kinetics at various running speeds.

Summarizing the present study, we observed differences in GM fascicle and SEE behavior, and the kinetics were different 
between the FFS and RFS. The fascicle length was shorter throughout the stance phase in the FFS than RFS, but the shortening velocity of the fascicle did not differ between the FFS and RFS. Interestingly, negative work done by the fascicle was slightly higher in the FFS than in the RFS, in accordance with our hypothesis. This indicates that the FFS is advantageous for obtaining energy from the elastic energy recoil in the propulsion phase of the stance. However, it would be disadvantageous for the use of the GM fascicle in the FFS because of its shorter length through the stance phase, compared with the RFS. The RFS may be a beneficial foot strike pattern for economical running due to its reduction in excessive muscle-tendon dynamics compared with the FFS.

\section{Acknowledgments}

The authors thank Dr Naokazu Miyamoto (Juntendo University, Japan) for his comments on this study and thank all participants for spending time and participating in the current study. They also thank Editage (www.editage. com) for English-language editing. This work was supported by the Joint Research Program of Juntendo University, Faculty of Health and Sports Science. The authors have no conflict of interest to disclose.

\section{References}

1. Hasegawa H, Yamauchi T, Kraemer WJ. Foot strike patterns of runners at the $15-\mathrm{km}$ point during an elite-level half marathon. $J$ Strength Cond Res. 2007;21(3):888-893. PubMed ID: 17685722 doi:10.1519/R-22096.1

2. Lieberman DE, Venkadesan M, Werbel WA, et al. Foot strike patterns and collision forces in habitually barefoot versus shod runners. Nature. 2010;463(7280):531-535. PubMed ID: 20111000 doi:10. 1038/nature 08723

3. Hanley B, Bissas A, Merlino S, Gruber AH. Most marathon runners at the 2017 IAAF World Championships were rearfoot strikers, and most did not change footstrike pattern. J Biomech. 2019;92:54-60. PubMed ID: 31147098 doi:10.1016/j.jbiomech. 2019.05.024

4. Larson P, Higgins E, Kaminski J, et al. Foot strike patterns of recreational and sub-elite runners in a long-distance road race. $J$ Sports Sci. 2011;29(15):1665-1673. PubMed ID: 22092253 doi:10. 1080/02640414.2011.610347

5. Gruber AH, Umberger BR, Braun B, Hamill J. Economy and rate of carbohydrate oxidation during running with rearfoot and forefoot strike patterns. J Appl Physiol. 2013;115(2):194-201. PubMed ID: 23681915 doi:10.1152/japplphysiol.01437.2012

6. Ogueta-Alday A, Rodríguez-Marroyo JA, García-López J. Rearfoot striking runners are more economical than midfoot strikers. Med Sci Sports Exer. 2014;46(3):580-585. PubMed ID: 24002340 doi:10. 1249/MSS.0000000000000139

7. Kulmala JP, Avela J, Pasanen K, Parkkari J. Forefoot strikers exhibit lower running-induced knee loading than rearfoot strikers. Med Sci Sports Exerc. 2013;45(12):2306-2313. PubMed ID: 23748735 doi:10.1249/MSS.0b013e31829efcf7

8. Valenzuela KA, Lynn SK, Mikelson LR, Noffal GJ, Judelson DA. Effect of acute alterations in foot strike patterns during running on sagittal plane lower limb kinematics and kinetics. J Sports Sci Med. 2015;14(1):225-232. PubMed ID: 25729311

9. Kuhman D, Melcher D, Paquette MR. Ankle and knee kinetics between strike patterns at common training speeds in competitive male runners. Eur J Sport Sci. 2016;16(4):433-440. PubMed ID: 26371382 doi:10.1080/17461391.2015.1086818
10. Prilutsky BI, Petrova LN, Raitsin LM. Comparison of mechanical energy expenditure of joint moments and muscle forces during human locomotion. J Biomech. 1996;29(4):405-415. PubMed ID: 8964770 doi:10.1016/0021-9290(95)00083-6

11. Hof AL, Van Zandwijk JP, Bobbert MF. Mechanics of human triceps surae muscle in walking, running and jumping. Acta Physiol Scand. 2002;174(1):17-30. PubMed ID: 11851593 doi:10.1046/j.1365201x.2002.00917.x

12. Farris DJ, Sawicki GS. Human medial gastrocnemius force-velocity behavior shifts with locomotion speed and gait. Proc Natl Acad Sci U S A. 2012;109(3):977-982. PubMed ID: 22219360 doi:10.1073/ pnas. 1107972109

13. Perl DP, Daoud AI, Lieberman DE. Effects of footwear and strike type on running economy. Med Sci Sports Exerc. 2012;44(7):13351343. PubMed ID: 22217565 doi:10.1249/MSS.0b013e318247989e

14. Hamill J, Gruber AH, Derrick TR. Lower extremity joint stiffness characteristics during running with different footfall patterns. Eur $J$ Sport Sci. 2014;14(2):130-136. PubMed ID: 24533519 doi:10.1080/ 17461391.2012.72824

15. Swinnen W, Hoogkamer W, Delabastita T, Aeles J, De Groote F, Vanwanseele B. Effect of habitual foot-strike pattern on the gastrocnemius medialis muscle-tendon interaction and muscle force production during running. J Appl Physiol. 2019;126(3):708-716. PubMed ID: 30629477 doi:10.1152/japplphysiol.00768.2018

16. Suzuki T, Ogane R, Yaeshima K, Kinugasa R. Forefoot running requires shorter gastrocnemius fascicle length than rearfoot running. $J$ Sports Sci. 2019;37(17):1972-1980. PubMed ID: 31032698 doi:10. 1080/02640414.2019.1610146

17. Yong JR, Dembia CL, Silder A, Jackson RW, Fredericson M, Delp SL. Foot strike pattern during running alters muscle-tendon dynamics of the gastrocnemius and the soleus. Sci Rep. 2020;10(1):5872. PubMed ID: 32245985 doi:10.1038/s41598-020-62464-3

18. Winter DA. Biomechanics and Motor Control of Human Movement. 4th ed. Hoboken, NJ: John Wiley \& Sons; 2009.

19. Delp SL, Loan JP, Hoy MG, Zajac FE, Topp EL, Rosen JM. An interactive graphics-based model of the lower-extremity to study orthopedic surgical-procedures. IEEE Trans Bio Med Eng. 1990; 37(8):757-767. doi:10.1109/10.102791

20. Hawkins D, Hull ML. A method for determining lower-extremity muscle tendon lengths during flexion extension movements. J Biomech. 1990;23(5):487-494. PubMed ID: 2373721 doi:10.1016/00219290(90)90304-L

21. Fukunaga T, Kubo K, Kawakami Y, Fukashiro S, Kanehisa H, Maganaris CN. In vivo behaviour of human muscle tendon during walking. Proc Biol Sci. 2001;268(1464):229-233. PubMed ID: 11217891 doi:10.1098/rspb.2000.1361

22. Fukunaga T, Roy RR, Shellock FG, et al. Physiological crosssectional area of human leg muscles based on magnetic-resonanceimaging. J Orthop Res. 1992;10(6):926-934. PubMed ID: 1403308 doi:10.1002/jor.1100100623

23. Kurokawa S, Fukunaga T, Fukashiro S. Behavior of fascicles and tendinous structures of human gastrocnemius during vertical jumping. J Appl Physiol. 2001;90(4):1349-1358. PubMed ID: 11247934 doi:10.1152/jappl.2001.90.4.1349

24. Ishikawa M, Pakaslahti J, Komi PV. Medial gastrocnemius muscle behavior during human running and walking. Gait Posture. 2007; 25(3):380-384. PubMed ID: 16784858 doi:10.1016/j.gaitpost.2006. 05.002

25. Hashizume S, Yanagiya T. A forefoot strike requires the highest forces applied to the foot among foot strike patterns. Sports Med Int Open. 2017;1(2):E37-E42. PubMed ID: 30539084 doi:10.1055/s0042-122017 
26. Stearne SM, Alderson JA, Green BA, Donnelly CJ, Rubenson J. Joint kinetics in rearfoot versus forefoot running: implications of switching technique. Med Sci Sports Exerc. 2014;46(8):1578-1587. PubMed ID: 24500531 doi:10.1249/MSS.0000000000000254

27. Arampatzis A, De Monte G, Karamanidis K, Morey-Klapsing G, Stafilidis S, Brüggemann GP. Influence of the muscle-tendon unit's mechanical and morphological properties on running economy. J Exp Biol. 2006;209(17):3345-3357. PubMed ID: 16916971 doi:10.1242/ jeb.02340

28. Kubo K, Miyazaki D, Tanaka S, Shimoju S, Tsunoda N. Relationship between Achilles tendon properties and foot strike patterns in longdistance runners. J Sports Sci. 2015;33(7):665-669. PubMed ID: 25277276 doi:10.1080/02640414.2014.962576

29. Williams DS, McClay IS, Manal KT. Lower extremity mechanics in runners with a converted forefoot strike pattern. J Appl Biomech. 2000;16(2):210-218. doi:10.1123/jab.16.2.210
30. Almonroeder T, Willson JD, Kernozek TW. The effect of foot strike pattern on Achilles tendon load during running. Ann Biomed Eng. 2013;41(8):1758-1766. PubMed ID: 23640524 doi:10.1007/s10439013-0819-1

31. Lyght M, Nockerts M, Kernozek TW, Ragan R. Effects of foot strike and step frequency on Achilles tendon stress during running. $J$ Appl Biomech. 2016;32(4):365-372. PubMed ID: 26955843 doi:10.1123/ jab.2015-0183

32. Daoud AI, Geissler GJ, Wang F, Saretsky J, Daoud YA, Lieberman DE. Foot strike and injury rates in endurance runners: a retrospective study. Med Sci Sports Exerc. 2012;44(7):1325-1334. PubMed ID: 22217561 doi:10.1249/MSS.0b013e3182465115

33. Sasaki K, Neptune RR. Muscle mechanical work and elastic energy utilization during walking and running near the preferred gait transition speed. Gait Posture. 2006;23(3):383-390. PubMed ID: 16029949 doi:10.1016/j.gaitpost.2005.05.002 